\title{
BMJ Open Protocol for the National Nurse Health Study (NNHS): a web-based ambispective cohort study
}

\author{
Lin Zhuo (1) , ${ }^{1}$ Heli Zhang, ${ }^{2}$ Rongmei Geng, ${ }^{2}$ Panfeng Wang, ${ }^{2}$ Lin Zeng (D) , 1 \\ Ying Che, ${ }^{3}$ Peng Wang, ${ }^{3}$ Pengfei Li, ${ }^{4}$ Tao Huang (D) , ${ }^{5}$ Baohua Li, ${ }^{2}$ Siyan Zhan (D) ${ }^{1,5}$
}

To cite: Zhuo L, Zhang $\mathrm{H}$, Geng R, et al. Protocol for the National Nurse Health Study (NNHS): a web-based ambispective cohort study. BMJ Open 2021;11:e049958. doi:10.1136/ bmjopen-2021-049958

- Prepublication history and additional supplemental material for this paper are available online. To view these files, please visit the journal online. (http://dx.doi.org/10.1136/ bmjopen-2021-049958).

$\mathrm{LZ}$ and $\mathrm{HZ}$ contributed equally.

Received 05 February 2021 Accepted 02 August 2021

Check for updates

(C) Author(s) (or their employer(s)) 2021. Re-use permitted under CC BY-NC. No commercial re-use. See rights and permissions. Published by BMJ.

${ }^{1}$ Research Center of Clinical Epidemiology, Peking University Third Hospital, Beijing, China ${ }^{2}$ Nursing Department, Peking University Third Hospital, Beijing, China

${ }^{3}$ Medical Examination Center, Peking University Third Hospital, Beijing, China

${ }^{4}$ Advanced Institute of Information Technology, Peking University, Hangzhou, Zhejiang, China

${ }^{5}$ Department of Epidemiology and Biostatistics, School of Public Health, Peking University, Beijing, China

Correspondence to Prof Baohua Li; lianglbh@126.com and Prof Siyan Zhan;

siyan-zhan@bjmu.edu.cn

\section{ABSTRACT}

Introduction The physical and mental health of nurses may significantly impact the entire medical care group and directly affect the quality of medical services. Due to the intense emotional involvement and often problematic working conditions that characterise their profession, nurses appear to be especially susceptible to a complex set of stressors with repercussions to their health. Several landmark studies of nurses have provided an abundance of evidence on risk factors that influence the health status of nurses. However, few studies have investigated the health status of nurses who work in high-intensity work environments in China. The National Nurse Health Study (NNHS) objective is to build an ambispective cohort to gather web-based information on early-life events, daily habits, occupational and environmental risk factors, and health outcomes of a specific subset of healthcare professionals of Chinese nurses.

Methods and analysis NNHS, which was developed at a tertiary hospital in Beijing, China, is a research initiative that enrolls registered nurses working at Peking University Third Hospital. A web-based self-administered system was designed to collect health-related data and link them with previous physical examination data. During the study period, participants with signed informed consent will be invited to annually repeat a similar procedure.

Ethics and dissemination The NNHS research protocol was approved by the Institutional Ethics Committee and provides promising data that contribute to the understanding of pathophysiological links between early-life events, body composition, gut microbiota, and inflammatory and metabolic risk profiles. Moreover, the combination of a user-friendly tool with the innovative purposes of the NNHS offers a remarkable resource to test hypotheses about mechanisms of diseases, including work stress, and further plan preventive programmes in public health.

Trial registration number The study was registered on Clinicaltrials.gov (https://clinicaltrials.gov/ct2/show/ NCT04572347) and the China Cohort Consortium (http:// chinacohort.bjmu.edu.cn/project/102/).

\section{INTRODUCTION}

With increasing demand and evolving scope, nurses constitute the largest healthcare provision group and play an increasingly important role in healthcare delivery. According to the

\section{Strengths and limitations of this study}

This study focuses on the long-term and stable comprehensive nurse health status (including mental health) and can assess the overall risks and benefits of lifestyle factors by evaluating them in relation to a range of mental disorders and other chronic conditions in a Chinese tertiary hospital.

- This study is the first ambispective cohort study via a web-based system of women with updated exposure assessment for a broad range of lifestyle factors, which is costless to develop and easy to outreach.

- More than 10 years of historical physical examination data linked with work shift administrative data lays a good foundation for the study.

- There are language-related limitations in the webbased system (only in Chinese).

- The generalisability of the study may be limited because of the limited sample size.

World Health Statistics Report, the number of nurses and midwives reached 29 million globally by 2017 , and estimates of upwards of one million additional nurses will be needed by $2020 .^{12}$ Even with this growth, which is closely related to the quality of care and patient safety, the nursing profession is still facing shortages. ${ }^{34}$ According to the WHO, the nursing deficit will reach 12.9 million by $20355^{56}$

The health status of nurses has garnered worldwide attention, especially when the nursing profession globally faces shortages, high turnover and inequitable healthcare distribution. ${ }^{7-9}$ Several landmark studies of nurses have increased our understanding of nurses' and women's health. Studies of this type include the oldest and most extensive nurse cohort study, which is the Nurse Health Study (NHS), and the following NHS II and NHS III, which made valuable contributions to our understanding of the risk factors for major chronic diseases in women. Moreover, its success highlighted the feasibility and 
advantages of recruiting nurses for long-term cohort studies of women's health. Furthermore, other countries such as Denmark, ${ }^{10}$ Japan, ${ }^{11}$ Australia, New Zealand, ${ }^{12}$ Thailand $^{13}$ and Korea ${ }^{14}$ took lessons from the NHS research type. Although numerous studies have identified factors that influence the nurse labour supply and demand, most of these studies were conducted in developed countries.

Chinese hospitals are witnessing a continuous increase in nurses. As of 2018, there were 4.1 million registered nurses in China compared with 30000 in the early 1950s. ${ }^{15}$ The number of nurses on a per capita basis has increased from 0.5 per 1000 population in 1980 to 2.7 per 1000 population in $2017,{ }^{16}$ which is well below the average of 9 of the Organization for Economic Cooperation and Development. ${ }^{17}$ Although it holds tremendous promise that the number of registered nurses in China will have reached 4.5 million by 2020 with a nurse-to-doctor ratio of $1: 1.25,{ }^{15}$ the heavy workload, low wages, and poor working conditions contribute to exacerbating the existing shortage of nurses. ${ }^{18}$ The physical and mental health of nurses may significantly impact the entire medical care group. Therefore, it directly affects the quality of medical services provided. ${ }^{19}$ In addition, nurses in China may carry a heavier workload than those in developed countries such as the US due to an unbalanced condition of the mind and body, which creates demands on the physiological and psychological state of nurses. ${ }^{20}$ At present, the most common methods to monitor nurse health in China are cross-sectional studies. For example, a previous study surveyed 27575 nurses in 136 hospitals across mainland China and showed that the overall mental health status of nurses was weak with enormous pressure on career development. ${ }^{21}$ Similarly, social and environmental factors such as diet and lifestyle play an essential role in developing major chronic diseases in nurses.

Considering the difficulties in prospectively organising a nurse cohort in China, long-term previous physical examination data are a precious resource for nurse health status research. In addition, web-based systems offer unique advantages compared with traditional printed questionnaire-based surveys, minimise errors and duplicated information, reduce costs, and provide the almost immediate construction of a database. ${ }^{22-27}$ The purpose of the current study was to build an ambispective cohort study of female nurses based on existing physical examination and nurse work schedule data and a webbased questionnaire system, which focuses on the effects of occupational, environmental and lifestyle risk factors on the health of Chinese women.

\section{Study objectives}

The primary objective is to assess the health status, including attention to mental health, and observe the changes in health status in nurses in a tertiary hospital in China in a long-term manner.

The secondary objectives are: (1) to study the relationship between factors such as lifestyle, social support, personnel management mode, and environmental exposure and the psychophysical health and career development of nurses; (2) to study the relationship between metabolic indices such as blood lipid biochemistry and women's health; (3) to formulate and develop a successful model for a future national nurse health cohort study.

\section{METHODS AND ANALYSIS \\ Study design}

This longitudinal ambispective cohort study combines physical examination data and nurse scheduling data with a web-based questionnaire for long-term follow-up. The study is registered on Clinicaltrials.gov (https://clinicaltrials.gov/ct2/show/NCT04572347) and the China Cohort Consortium (http://chinacohort.bjmu.edu.cn/ project/102/). Moreover, the study was planned to start on 1 July 2021 and complete the first follow-up on 1 September 2021.

\section{Participants and study site}

According to a previous study on nurses in 136 hospitals in China, ${ }^{28} 52.5 \%$ of female nurses reported a low score on mood. A sample size of 401 produces a two-sided 95\% CI with a width of 0.100 when the sample proportion is 0.525 . Peking University Third Hospital, which is a famous tertiary hospital in China with a high-quality information construction foundation, employs approximately 2000 nurses with a separation rate of $0.005 \%$. In addition, nearly 10 years of electronic, physical examination data can be used to extend the cohort's length.

We will recruit participants from Peking University Third Hospital in Beijing, China, through cluster sampling. Female registered nurses and licensed practical nurses who provided informed consent will be included in this study. The exclusion criteria are student nurses, training nurses, and those who are unwilling to participate in research. Specific exclusion and inclusion criteria regarding study data will be determined for each study in the future.

\section{Recruitment and follow-up}

Eligible participants will log onto the online survey system using their name and nurse registration number. On agreeing to participate in the online survey, potential participants will be provided with a full explanation of the study and a paper-based informed consent form. Informed consent includes acknowledgement of (1) voluntary study participation; (2) the use of survey data (but not personal information) for research purposes, confidentiality, and sharing of the study results; (3) safeguarding individual information (eg, nurse registration numbers, e-mail addresses, and contact phone numbers) for follow-up invitation and disease ascertainment; (4) permission to access previous physical examination data; (5) permission to undertake subsequent follow-up regarding disease occurrence via links with physical examination data; (6) the right to refuse or withdraw at any 
Table 1 Summary table of parameters and measurements

\begin{tabular}{|c|c|c|c|c|}
\hline \multicolumn{2}{|l|}{ Domain } & Parameter & Instrument/technique & Assessing person \\
\hline \multicolumn{2}{|c|}{$\begin{array}{l}\text { Demographic and socioeconomic } \\
\text { characteristics }\end{array}$} & $\begin{array}{l}\text { Birthdate } \\
\text { Birthplace } \\
\text { Marital status } \\
\text { Fertility status } \\
\text { Education } \\
\text { Menstrual cycle } \\
\text { Family history }\end{array}$ & $\begin{array}{l}\text { Self-administered web-based } \\
\text { questionnaire }\end{array}$ & Participant \\
\hline $\begin{array}{l}\text { Occupation } \\
\text { characteristics }\end{array}$ & General information & $\begin{array}{l}\text { Working start date } \\
\text { Nursing qualification } \\
\text { Employment status } \\
\text { Employment position } \\
\text { Income } \\
\text { Job transition since graduation }\end{array}$ & $\begin{array}{l}\text { Self-administered web-based } \\
\text { questionnaire }\end{array}$ & Participant \\
\hline \multirow[t]{3}{*}{ Health status } & Pysical health & $\begin{array}{l}\text { History and current illness } \\
\text { Medication used in the past } 30 \text { days } \\
\text { Hospitalisation during the past } 12 \text { months }\end{array}$ & $\begin{array}{l}\text { Self-administered web-based } \\
\text { questionnaire }\end{array}$ & Participant \\
\hline & Mental health status & $\begin{array}{l}\text { National Aeronautics and Space Administration Task } \\
\text { Load Index, NASA-TLX } \\
\text { Symptom Checklist 90, SCL-90 } \\
\text { Chinese version Career Success Scale } \\
\text { Nursing Work Index-Revised, NWI-R }\end{array}$ & $\begin{array}{l}\text { Self-administered web-based } \\
\text { questionnaire }\end{array}$ & Participant \\
\hline & Anthropometry & $\begin{array}{l}\text { Height } \\
\text { Weight } \\
\text { Body mass index (BMI) } \\
\text { Blood pressure } \\
\text { Heart rate } \\
\text { Vision } \\
\text { Hearing } \\
\text { Pain check } \\
\text { Body fat composition }\end{array}$ & Physical examination & Nurses \\
\hline
\end{tabular}

time; (7) no direct benefit for any participant from participating in the research. Participants will receive annual follow-up by a web-based questionnaire booklet, and data will be extracted from the physical examination system.

\section{Data collection and management}

The content validity of the questionnaire booklet was assessed by two nurses from the Peking University Third Hospital. They were asked whether the questions were suitable for the participants and whether any essential concepts were missing from the inventory of questions of the booklet. Subsequently, the web-based system was pilot tested, including the convenience and recognizability of the system. The questionnaire booklet in the baseline survey has four main sections (table 1): (1) demographic and socioeconomic characteristics (such as date of birth, gender, name, work department, previous medical history, current medical treatment, and reproductive health information). Reproductive health information includes information on menarche, pregnancy, gestational diabetes and hypertension, oral contraceptive use, and female hormone use; (2) lifestyle characteristics (including behaviours such as smoking, drinking, sleep duration, obstructive sleep apnoea risk, snoring, and physical activity); (3) occupation characteristics (general 
information and work intensity); (4) health status (mental health status, anthropometry). The follow-up survey repeats the occupation and mental health status sections. Related data from physical examination data, laboratory test results, and nursing schedule information systems will be directly extracted from the system via employee numbers. Online questionnaire data will be automatically saved on the server at the secure internet data centre, which is designated by the NNHS IT team. The intrusion prevention system has periodic management and backup. Processed data will be annually cleaned and analysed by the analysis team after every follow-up. If any error occurs in the data management, the NNHS data team will solve the problem and report it to the steering committee. The Chinese version of the survey form can be found in online supplemental file 1 .

\section{Data protection}

In this study, retrospectively and prospectively collected sensitive health data will be assessed. Other sensitive data (eg, religious, political, social security, or administrative data) will not be assessed. Data will be handled according to the Chinese data protection regime.

\section{Outcomes}

The participants are made aware of their health status when their physical examination results are complete, and they often receive advice and suggestions from health professionals. Therefore, our cohort studies are not natural history cohort studies by nature. Some participants receive treatment in hospitals, and some receive further health guidance because of the results of their check-ups.

In a series of study scenarios, we will consider various conditions and aetiologies as the primary and secondary outcomes according to the study objectives. Currently, we focus on the psychological impacts on nurses: (1) influence of night shifts on the sleep quality of nurses (taking insomnia and difficulty falling asleep as outcomes); (2) influence of a hierarchical management system on the career development of nurses (taking punishment and promotion as outcomes); (3) influence of family social support on the occupational stability of nurses (taking resignation, post-transfer, and sick leaves as outcomes).

\section{Statistical consideration}

Statistical methods will be selected according to the study question under investigation. For cross-sectional analyses, data will be described using SD and medians with IQR for continuous variables and frequencies and proportions for categorical variables. The differences of groups will be compared using a t-test or for normally distributed continuous variables and non-parametric tests for nonnormally distributed continuous variables; $\chi^{2}$ testing will be used for categorical variables. Subgroup analyses will be performed to discover the differences between age groups, departments, advanced nursing roles and working hours. Linear and/or logistic regression models with or without adjustment for potential confounders will be used whenever appropriate. To analyse longitudinal data, survival analyses, including Kaplan-Meier curves and Cox proportional hazards models, will be used whenever appropriate. To control for possible bias and confounding factors in this cohort study, we will use matching or take exposure as a time-varying covariate in the analysis stage and incorporate it into the model analysis. For all tests, a $\mathrm{p}$ value $<0.05$ will be considered significant. Analyses will be performed using SAS statistical software (V.9.4).

A sensitivity analysis was performed to assess the potential impact of missing data. If the missing rate of critical parameters (such as occupation characteristics and laboratory test results extracted from the HIS) is over $20 \%$, the record will be deleted.

\section{ETHICS AND DISSEMINATION}

Informed consent will be voluntarily obtained from each study participant before enrolment in the study. It will include access to previous physical examination data of the participants and link the data with the nursing schedule information system. According to the local regulations, the baseline survey participants and follow-up study will not receive any subsidies from the study. The ethical committee and regulatory authorities will receive interim reports and be informed about the stopping/ ending of the study in agreement with local requirements. The study will be performed following the protocol and with principles enunciated in the current version of the Declaration of Helsinki, the guidelines of Good Clinical Practice issued by the International Conference on Harmonisation of Technical Requirements for Registration of Pharmaceuticals for Human Use, Chinese law, and the Chinese regulatory authority's requirements. The findings of the study will be disseminated through peerreviewed journals and national and international conference presentations.

Contributors $\mathrm{LZ}$ and $\mathrm{HZ}$ are joint first authors. BL obtained funding. PL and SZ had full access to all of the data in the study and take responsibility for the integrity of the data and the accuracy of the data analysis. LZ, BL and SZ conceived of and designed the study. LZ, HZ, RG, Panfeng Wang, LZ, YC and PW participated in the elaboration of the protocol. PL and LZ programmed and tested the web-based system. LZ drafted the manuscript. All authors have read and approved the final manuscript.

Funding This study is supported by grants from the National Natural Science Foundation of Ningxia Province (2020AAC03501) and the Cohort Construction Project of Peking University Third Hospital (LM2020444).

Competing interests None declared.

Patient consent for publication Not required.

Ethics approval The NNHS study protocol and study documentation, including all questionnaires and informed consent, were approved by the Peking University Third Hospital Medical Science Research Ethics Committee (2020 316-01).

Provenance and peer review Not commissioned; externally peer reviewed.

Supplemental material This content has been supplied by the author(s). It has not been vetted by BMJ Publishing Group Limited (BMJ) and may not have been peer-reviewed. Any opinions or recommendations discussed are solely those of the author(s) and are not endorsed by BMJ. BMJ disclaims all liability and responsibility arising from any reliance placed on the content. Where the content 
includes any translated material, BMJ does not warrant the accuracy and reliability of the translations (including but not limited to local regulations, clinical guidelines, terminology, drug names and drug dosages), and is not responsible for any error and/or omissions arising from translation and adaptation or otherwise.

Open access This is an open access article distributed in accordance with the Creative Commons Attribution Non Commercial (CC BY-NC 4.0) license, which permits others to distribute, remix, adapt, build upon this work non-commercially, and license their derivative works on different terms, provided the original work is properly cited, appropriate credit is given, any changes made indicated, and the use is non-commercial. See: http://creativecommons.org/licenses/by-nc/4.0/.

\section{ORCID iDs}

Lin Zhuo http://orcid.org/0000-0001-9188-702X

Lin Zeng http://orcid.org/0000-0001-8707-5854

Tao Huang http://orcid.org/0000-0002-0328-1368

Siyan Zhan http://orcid.org/0000-0001-7252-5349

\section{REFERENCES}

1 Slattery MJ, Logan BL, Mudge B, et al. An undergraduate research fellowship program to prepare nursing students for future workforce roles. J Prof Nurs 2016;32:412-20.

2 World Health Organization. The 2018 update, global health workforce statistics, 2018. Available: http://www.who.int/hrh/statistics/hwfstats/ [Accessed 29 Apr 2020].

3 Marć M, Bartosiewicz A, Burzyńska J, et al. A nursing shortage - a prospect of global and local policies. Int Nurs Rev 2019;66:9-16.

4 Chan ZCY, Tam WS, Lung MKY, et al. A systematic literature review of nurse shortage and the intention to leave. J Nurs Manag 2013;21:605-13.

5 Global Health Workforce Alliance and World Health Organization. A universal truth: no health without a workforce 2013. Available: https://www.who.int/workforcealliance/knowledge/resources/GHWAa_universal_truth_report.pdf?ua=1 [Accessed 6 May 2019].

6 World Health Organization. Global strategy on human resources for health: workforce 2030, 2015. Available: https://www.who.int/hrh/ resources/pub_globstrathrh-2030/en/ [Accessed 28 Apr 2020].

7 Vetter C, Devore EE, Wegrzyn LR, et al. Association between rotating night shift work and risk of coronary heart disease among women. JAMA 2016;315:1726-34.

8 Schernhammer ES, Feskanich D, Liang G, et al. Rotating night-shift work and lung cancer risk among female nurses in the United States. Am J Epidemiol 2013;178:1434-41.

9 Lawson CC, Johnson CY, Chavarro JE, et al. Work schedule and physically demanding work in relation to menstrual function: the nurses' health study 3. Scand J Work Environ Health 2015;41:194-203.

10 Sanderson DM, Ekholm O, Hundrup YA, et al. Influence of lifestyle, health, and work environment on smoking cessation among Danish nurses followed over 6 years. Prev Med 2005;41:757-60.
11 Hayashi K, Mizunuma H, Fujita T, et al. Design of the Japan nurses health study: a prospective occupational cohort study of women's health in Japan. Ind Health 2007;45:679-86.

12 Turner C, Bain C, Schluter PJ, et al. Cohort Profile: The Nurses and Midwives e-Cohort Study--a novel electronic longitudinal study. Int J Epidemiol 2009;38:53-60.

13 Sawaengdee K, Tangcharoensathien V, Theerawit T, et al. Thai nurse cohort study: cohort profiles and key findings. BMC Nurs 2016;15:10.

14 Kim O, Ahn Y, Lee H-Y, et al. The Korea nurses' health study: a prospective cohort study. J Womens Health 2017;26:892-9.

15 Ministry of Health of the People's Republic of China. Chinese nursing development plan (2016-2020). Chin J Nurs 2017;17:1-5.

16 National Bureau of Statistics of China. China statistical Yearbook 2018. Beijing, China: China Statistics Press, 2018.

17 OECD. Health workforce policies in OECD Countries-Right jobs, right skills, right places. Paris: OECD Publishing, 2016.

18 Yang J-S, Hao D-J. Dilemmas for nurses in China. Lancet 2018;392:30.

19 Adler NR, Adler KA, Grant-Kels JM. Doctors' mental health, burnout, and suicidality: professional and ethical issues in the workplace. $J$ Am Acad Dermatol 2017;77:1191-3.

20 Lu D-M, Sun N, Hong S, et al. Occupational stress and coping strategies among emergency department nurses of China. Arch Psychiatr Nurs 2015;29:208-12.

21 Shichao W, Yinuo W, Jing M. Self-evaluation and influential factors of mental health of nurses in 136 III level public hospitals. Health Economics Research 2019;36:62-5.

22 WCJBrm S. Instruments,. worldwide web survey research: benefits, potential problems, and solutions. Behav Res Meth Instrum Comput 1997;29:274-9.

23 Bälter KA, Bälter O, Fondell E, et al. Web-Based and Mailed questionnaires: a comparison of response rates and compliance. Epidemiology 2005;16:577-9.

24 Touvier M, Méjean C, Kesse-Guyot E, et al. Comparison between web-based and paper versions of a self-administered anthropometric questionnaire. Eur J Epidemiol 2010;25:287-96.

25 Hirsch O, Hauschild F, Schmidt MH, et al. Comparison of web-based and paper-based administration of ADHD questionnaires for adults. $J$ Med Internet Res 2013;15:e47.

26 Folchetti LGD, Silva ITda, Almeida-Pititto Bde, et al. Nutritionists' Health Study cohort: a web-based approach of life events, habits and health outcomes. BMJ Open 2016;6:e012081.

27 Mattsson S, Olsson EMG, Carlsson M, et al. Identification of anxiety and depression symptoms in patients with cancer: comparison between short and long web-based questionnaires. J Med Internet Res 2019;21:e11387.

28 Shi-chao W, Yi-nuo W, Jing M. Self-evaluation and influential factors of mental health of nurses in $136 \mathrm{III}$ level public hospitals. Health Economics Research 2019;8:62-5. 